\title{
Waist Circumference is a Better Predictor of Recurrent Pregnancy Loss When Compared to Body Mass Index among Nigerian Women
}

\author{
Ashiru Hassan Yusuf ${ }^{* *}$, Abubakar Shehu Akuyam ${ }^{2}$, \\ Murdakai Tanko ${ }^{1} \&$ Wilson Oliver Hamman ${ }^{1}$ \\ ${ }^{1}$ Department of Anatomy, \\ Faculty of Basic Medical Sciences, \\ Ahmadu Bello University Zaria \\ ${ }^{2}$ Department of Medical Laboratory Sciences, \\ Faculty of Allied Health Sciences, \\ Ahmadu Bello University Zaria \\ Email: ahyusuf.ana@buk.edu.ng
}

\begin{abstract}
Recurrent Pregnancy loss is a common obstetrics condition with severe physical and psychological consequences to the patients and attending physicians as well. It affects 1-5\% of women and up to $50 \%$ has no identifiable etiology. Obesity is one of the independent risk factor of this condition; however, the evidence of this association is conflicting. This study aims to further determine that obese women with higher visceral fat are at increased risk of Recurrent Pregnancy Loss. The study was a cross-sectional case-control at a public referral hospital in Kano state Nigeria where 116 women with recurrent pregnancy loss were compared to a comparison control group consisting of 116 normal women. In addition to their socio-demographics, weight, height, BMI, waist circumference, and Hip circumference were collected. It was discovered that Obese BMI range and High-risk value for WC were significantly higher odds of recurrent pregnancy loss (OR, 1.96; 95\% CI, 1.37-2.46; OR, 4.61 95\% CI, 3.458- 5.696 respectively) Logistic regression analysis showed that the most important factor predicting the occurrence of Recurrent pregnancy loss was waist circumference. The receiver operator characteristic (ROC) curve showed that WC has a considerably higher area under the curve (AUC) and BMI had the least AUC value. This study concludes that waist circumference may be superior to $B M I$ as a risk factor of Recurrent Pregnancy Loss.
\end{abstract}

Keywords: Recurrent Pregancy Loss, BMI, Waist Circumference.

\section{INTRODUCTION}

Recurrent Pregnancy Loss (RPL) as an obstetrics illness with severe psychological complications including, post-traumatic stress disorders, depression, pregnancy-related anxiety, irritability, excessive fatigue, fear, sleep disorders and lack of concentration (Tavoli et al., 2018). It is defined as loss of two or more pregnancies before the 24 weeks gestation affecting 1-5\% of all couples. (Aruna et al., 2011; ESHRE, 2017). In addition to its effect on the patients, RPL is equally distressing to clinicians simply because despite a detailed clinical evaluation, no apparent etiology is elicited in up to $40-50 \%$ of all cases (Ford and Schust,

\footnotetext{
*Author for Correspondence
} 
2009; Hong and Marren, 2018). As the etiology remains a mystery, researchers focus on the risk factors of RPL, especially the modifiable ones. Some of the most studied risks of RPL include Obesity, cigarette smoking, excessive use of caffeine, alcohol consumption, advanced maternal and paternal age and family history of miscarriage (Lashen et al., 2004; Boots and Stephenson, 2011; sagiura-Ogasawara et al., 2015).

Obesity is a recognized obstetric risk factor that has been linked to adverse pregnancy outcomes including higher risks of spontaneous and recurrent pregnancy loss (Boots and Stephenson, 2011). One study explained that an obese adolescent has three to fold higher risk of miscarriage and unexplained foetal deaths and even primary infertility in their reproductive life (Polotsky et al., 2010). However, the mechanism to back this relationship is still debated. Since RPL is generally considered a very challenging reproductive health problem, several researchers have made numerous attempts to explain its pathophysiology. The major reason has been the assumption that obesity generally affects female reproduction negatively due to ectopic adipose tissue deposition, greater oxidative stress, and low grade chronic inflammation (Longo et al., 2019). The obese women have also been shown to have Hormonal imbalance involving the Hypothalamus-pituitary hyperactivity (HPA), abnormally high serum LH level with consequent and premature ovulation and luteal phase defects (Leeners et al., 2017).

The great number of obese women with successful reproductive history has puzzled researchers over the years. One big unanswered question is how some obese women get to have successful reproductive life while others do not. This research therefore proposed that obese women with unhealthy visceral fat are more likely to experience poor obstetric outcome. Evidence has shown that visceral obesity in women is of importance and may be the major underlying risk factor for all obesity related complications (Leeners et al., 2017). This is because, in women of reproductive age, estrogen and estrogen signaling generally promotes subcutaneous adipose tissue deposition along the gluteo-femoral region and at the time prevents visceral adiposity (Palmer and Clegg, 2015). We therefore hypothesized that irrespective of BMI; excessive visceral adiposity is a pointer to reproductive dysfunction and may predict higher risk of RPL. The objective of the study was to compare the predictive powers of BMI and other visceral adiposity markers for occurrence of recurrent pregnancy loss. This research will to compare the relative risk of BMI to other measures of visceral adiposity with regards to RPL.

\section{MATERIALS AND METHODS}

\section{Study design"}

The study was a hospital-based, conducted at Murtala Muhammad Specialists Hospital and Muhammad Abdullahi Wase Teaching hospital in the metropolis of Kano State, Nigeria. The Hospitals are the major referral centers where cases of RPL across Kano State converge to see the specialists. The hospitals are located within the metropolis which is on latitude $12^{\circ} 021 \mathrm{~N}$, longitude $08 \mathrm{\circ} 301 \mathrm{E}$ in the north-western region of Nigeria (Fig.3.1) (Ki - Zerbo, 1998). Kano is the most populous state in Nigeria with a population of 9.4 million, and 499 square kilometers Kano state consists of 44 local government areas. The major inhabitants of Kano are of Hausa and Fulani ethnic groups with minorities representing virtually all tribes in Nigeria and a minute fraction of people from the Niger Republic, Ghana, and other nationals. (Dan-Asabe, 1991). 


\section{Ethical Consideration}

An ethical clearance to conduct the study was obtained from the research ethics committee of the Kano state ministry of health. Informed consent was obtained from each adult participant before.

Participant were women aged 16 to 45 years with two or more previous history of pregnancy loss (RPL) and another group of women who were apparently normal Participants were selected from the Gynecology clinic of the selected hospitals. A proforma was used to record the participant's age, ethnicity, location of birth, and history of miscarriage, and relevant family history.

Variables in the study were socio-demographic characteristics, number of miscarriage, body weight, height, waist and hip circumferences. Participants were selected randomly using the systematic sampling technique with sampling frame of 690 and interval of 2 . For the women with RPL, all those patients who were on the RPL were recruited.

Measurements: each patient was privately examined using a mobile hospital screen cover. Height was measured to the nearest $0.1 \mathrm{~cm}$ as the vertical distance between the standing surface and the vertex of the head with the participant standing upright without shoes using a stadiometer (Price et al., 2006). The weight was measured in kilograms using a digital weighing scale while the subject is in light clothing. The body mass index was calculated using the formula:

$\mathrm{BMI}=\left(\mathrm{wt}(\mathrm{kg}) / \mathrm{H}^{2}(\mathrm{~m})\right)$

Where wt =weight; $\mathrm{H}=$ height (Mueller et al., 2004).

Waist circumference (WC) was measured in centimeters with a non-stretchable tape placed horizontally over the abdomen at the narrowest point between the $12^{\text {th }}$ rib and the iliac crest (Lean et al., 1995). The hip circumference (HC) was measured while the subject was standing erect with the feet close together. The measuring tape was passed around the point with the maximum protuberance of the buttocks (Hsieh and Yoshinaga., 1999).

The ratio of WC and HC was recorded as waist-hip ratio (WHR).

Abdominal obesity indices were categorized as "normal" or "abnormal" based on the National Cholesterol Education Program (NCEP) reference values. Where WC was considered normal when it $<88 \mathrm{~cm}$ for females, and WHR was considered normal if $<0.85$ in females (NCEP, 2002).

\section{Statistical analyses}

Bias was eliminated by double blinding where the research assistants who selected participants were not the ones who took the measurement. Each measurement was repeated and the average was recorded to reduce observer error.

The study size involved 116 women with history of RPL and same number of controls.

Data was summarized as mean \pm standard deviations of the samples. Chi-square was used to test the relationship between the occurrence RPL with BMI and abdominal adiposity indices (WC, HC and WHR). Pearson's correlation was used to find the relationship between $\mathrm{BMI}$ and WC, HC and WHR. Logistic regression was used to evaluate the predictive power of the measured variables SPSS version 26 was used for statistical analyses and $p<0.05$ was set as level of significance. 


\section{RESULTS}

In this study it was shown that age was a significant predictor of RP and women age 35 years were more likely to have RPL. Similarly, women whose male partners are aged 45 years and above were more likely to have the condition. Also, women with family history of RPL and excessive intake of caffeine were more likely to have RPL. However no association was observed with tribe and religion as shown in (Table 1)

Also, (Table 2) showed that women with RPL have significantly higher mean BMI, WC, HC, WHR and blood pressure measurements. It was also observed that obese women according to BMI were 1.96 time more likely to have RPL compared to their lean counterparts. Similarly, women with high risk measurement of WC are 4.61 more likely to have RPL compared to those with normal range as shown in (table 3). The cuf off value BMI, WC and WHR above which the risk of RPL was seen to progressively increase was shown to be 27.01, 79.5 and 0.78 respectively as shown in figure 1 and table 4 respectively.

Table 1. Socio-demographic characteristics

\begin{tabular}{|c|c|c|c|c|c|c|}
\hline \multicolumn{2}{|l|}{ Variable } & \multirow{2}{*}{$\begin{array}{l}\text { Controls } \\
77\end{array}$} & \multirow{2}{*}{$\begin{array}{l}\text { Cases } \\
63\end{array}$} & \multirow{3}{*}{$\begin{array}{l}x^{2} \\
3.853\end{array}$} & OR $(95 \%$ CI $)$ & \multirow{3}{*}{$\begin{array}{l}\text { p-value } \\
0.040^{*}\end{array}$} \\
\hline Maternal & $16-34$ & & & & $1.8(1.43-2.03)$ & \\
\hline & $>35$ & 40 & 52 & & & \\
\hline \multirow{2}{*}{$\begin{array}{l}\text { Paternal } \\
\text { Age (years) }\end{array}$} & Normal & 31 & 19 & 3.912 & $1.68 \quad(1.012-4.47)$ & $0.045^{*}$ \\
\hline & Advanced & 86 & 96 & & & \\
\hline \multirow[t]{3}{*}{ Tribe } & Hausa & 107 & 94 & 3.786 & $0.93 \quad(0.43-2.03)$ & 0.143 \\
\hline & Yoruba & 6 & 14 & & & \\
\hline & Igbos & 3 & 8 & & & \\
\hline \multirow[t]{2}{*}{ Religion } & Islam & 113 & 105 & 0.628 & $0.34 \quad(0.015-4.12)$ & 0.428 \\
\hline & Christianity & 3 & 11 & & & \\
\hline \multirow[t]{2}{*}{ Family Hx } & $\mathrm{NO}$ & 112 & 98 & 10.043 & $1.51 \quad(1.10-4.65)$ & $0.002^{*}$ \\
\hline & YES & 4 & 18 & & & \\
\hline \multirow{2}{*}{$\begin{array}{l}\text { Household } \\
\text { hx of RPL }\end{array}$} & $\mathrm{NO}$ & 116 & 111 & 2.519 & $0.64 \quad(0.15-3.12)$ & 0.113 \\
\hline & YES & 0 & 5 & & & \\
\hline \multirow{2}{*}{$\begin{array}{l}\text { Excess } \\
\text { Caffeine }\end{array}$} & NO & 112 & 97 & 11.287 & $1.81 \quad(1.2-4.65)$ & $0.001^{*}$ \\
\hline & YES & 4 & 18 & & & \\
\hline
\end{tabular}


Table 2: Anthropometric characteristics of the study participants

\begin{tabular}{lllllll}
\hline & Controls & & Cases & & \\
Variable & Mean & Min-Max & Mean & Min-Max & $t$ & p-Value \\
\hline Wt.(kg) & $60.15 \pm 11.79$ & $47.9-93.4$ & $63.95 \pm 8.75$ & $47.90-93.40$ & 1.27 & $0.021^{*}$ \\
Ht(m) & $1.58 \pm 0.06$ & $1.5-1.79$ & $1.59 \pm 0.06$ & $1.47-1.79$ & 0.60 & 0.548 \\
BMI (kg/m2) & $24.89 \pm 4.59$ & $15.98-35.58$ & $27.49 \pm 3.46$ & $16.98-35.59$ & 3.48 & $0.020^{*}$ \\
WC(cm) & $79.43 \pm 10.25$ & $66-118$ & $86.92 \pm 6.63$ & $66-98$ & 3.61 & $0.010^{*}$ \\
HC (cm) & $96.44 \pm 8.29$ & $78-115$ & $89.95 \pm 6.68$ & $79-114$ & -5.55 & $0.000^{*}$ \\
WHR & $0.84 \pm 0.06$ & $0.7-1.12$ & $0.89 \pm 0.03$ & $0.78-1.12$ & 6.12 & $0.000^{*}$ \\
NC(cm) & $34.01 \pm 1.99$ & $31-39.67$ & $35.30 \pm 2.12$ & $27-37$ & -0.25 & 0.806 \\
SBP(mmHg) & $128.28 \pm 12.26$ & $90-160$ & $112.80 \pm 8.66$ & $90-130$ & 4.51 & $0.000^{*}$ \\
DBP(mmHg) & $69.25 \pm 8.56$ & $55-90$ & $72.46 \pm 9.52$ & $50-90$ & 10.68 & $0.000^{*}$ \\
\hline
\end{tabular}

BMI: body mass index, WC: waist circumference, HC: hip circumference, WHR: waist-to-hip ratio, NC: Neck circumference DBP: diastolic blood pressure, SBP: systolic blood pressure

Table 3: Relationship between BMI, WC, WHR and RPL

\begin{tabular}{lllllll}
\hline Variable & Categories & Cases & Controls & $\chi^{\mathbf{2}}$ & OR (95\% CI) & p-value \\
\hline BMI & Normal & 50 & 44 & 9.97 & $1.96(1.37-2.246)$ & $0.000^{*}$ \\
& Overweight & 33 & 66 & & & $0.000^{*}$ \\
& Obese & 10 & 6 & & & 0.919 \\
WC & Normal & 26 & 83 & 31.39 & $4.61(4.458-35.696)$ & \\
& High risk & 67 & 33 & & & 0.239 \\
WHR & Normal & 77 & 113 & 14.786 & $1.09(0.203-5.858)$ & \\
& High risk & 16 & 3 & & & $0.002(0.000-22.06)$ \\
SBP & Normal & 116 & 81 & 40.000 & & \\
& Raised & 6 & 35 & & & \\
DBP & Normal & 107 & 89 & 1.0580 & $0.186(0.023-1.500)$ & \\
& Raised & 9 & 27 & & &
\end{tabular}

BMI: body mass index, WC: waist circumference, HC: hip circumference, WHR: waist-to-hip ratio, DBP: diastolic blood pressure, SBP: systolic blood pressure 


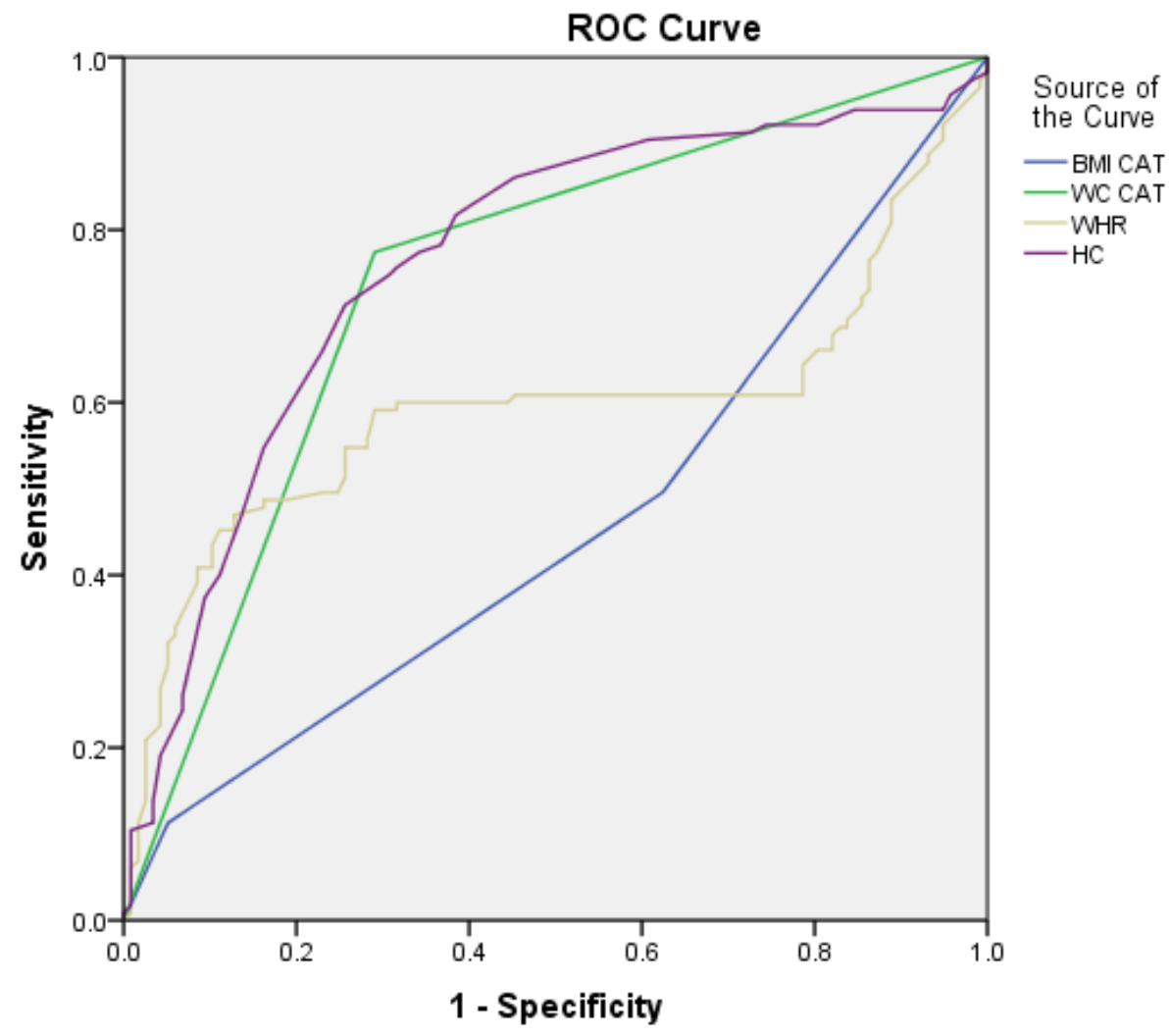

Diagonal segments are produced by ties.

Figure 1. cut off values of BMI, WC, HC and WHR for occurrence of RPL

Table 4: cut off values of BMI, WC, HC and WHR for occurrence of RPL

\begin{tabular}{|c|c|c|c|c|c|c|c|c|c|}
\hline \multirow[t]{2}{*}{ Variable } & \multirow[t]{2}{*}{ AUC } & \multirow[t]{2}{*}{ Std. Error } & \multirow[t]{2}{*}{ Sig. ${ }^{b}$} & \multicolumn{6}{|c|}{$95 \% \mathrm{CI}$} \\
\hline & & & & Lower & Upper & cut off & sensitivity & specificity & YI \\
\hline BMI & 0.438 & 0.041 & 0.123 & 0.357 & 0.519 & 27.01 & 0.759 & 0.241 & 1 \\
\hline WC & 0.708 & 0.039 & 0.000 & 0.632 & 0.784 & 79.5 & 0.891 & 0.724 & 1.615 \\
\hline HC & 0.745 & 0.036 & 0.000 & 0.677 & 0.816 & 82.11 & 0.75 & 0.379 & 1.129 \\
\hline WHR & 0.649 & 0.062 & 0.102 & 0.527 & 0.771 & 0.78 & 0.859 & 0.621 & 1.48 \\
\hline
\end{tabular}

BMI: body mass index, WC: waist circumference, HC: hip circumference, WHR: waist-to-hip ratio

\section{DISCUSSION}

The finding in study that advanced maternal age were significant predictors of RPL is similar to what was documented in the literature by (Nybo-Anderson et al., 2000; du-Fossé, et al. 2020 and Frick, 2021). The reason behind this risk was been shown to be the progressive loss of DNA integrity in both males and females (Zhu et al., 2005; Ozawa et al., 2019). Similarly, high caffeine intake was equally shown to be a significant predictor of RPL in previous studies (Pollack et al., 2010). Likewise the positive family history of RPL which generally suggest genetic predisposition (Woolmer et al., 2020). 
The findings in this study clearly suggested the mean BMI was significantly higher among women with RPL compared to the normal ones (Table 2). When categorized according to the WHO classification of BMI, BMI was found to be significantly associated with RPL and those with obese range of BMI are 2 times more likely to have RPL compared to lean individuals. This finding is similar to what was obtained by Lashen et al, (2004) when they reviewed clinical data from over 16,000 women and concluded that women with an obese range of $\mathrm{BMI}$ are $20 \%$ more likely to have RPL compared to overweight, normal-weight individuals $(3.5,95 \%$ CI 1.03-12.01 P = 0.04). Additionally, a systematic review by (Boots and Stephenson, 2011) of over 28,000 women in the United States revealed that the odds of RPL among the obese were almost 4 folds compared to the normal weight individuals (OR: 3.51; 95\% CI, 1.03 to 12.01). In a somewhat varied result, Metwally et al, (2010) showed that extremes of BMI i.e. obese and underweight are collectively at increased risk of RPL compared to normal and overweight individuals. However, they equally stressed the role of an obese range of BMI to approach 4 folds (OR-3.98; 95\% CI, 1.06-14.92). While stressing the higher chance of repeat miscarriage among the Asian population, Lo et al, (2012) equally stated that the risk of RPL among the obese was significantly higher than lean women by (OR 1.73; 95\% CI 1.06 - 2.83). A more recent data showed that RPL exclusively related obese BMI range with (OR, 1.75; 95\% CI, 1.24-2.47; $\mathrm{P}=0.001)$ (Cavalcante et al., 2019).

Although, these studies have consistently proved that the obese range of BMI is indeed an established risk factor for RPL, the reason behind it is still debated. Li et al, (2000) conducted an in-depth risk assessment analysis among obese women with RPL and discovered that $8 \%$ had raise serum LH levels, which predispose them to premature LH surge and ovulation of poor quality oocytes and hence poor quality embryos' that would be eventually miscarried. Also, $7.8 \%$ were found to have evidence of PCOS. The study also found that free androgen index (FAI) which a ratio of total serum testosterone to the sex hormone-binding globulin (SHBG) was equally elevated in about $14.6 \%$ of obese women with RPL. Another finding of the study was low mid-luteal phase progesterone among $17.4 \%$ of patients, poorly developed endometrium among 21.1\%. (Li et al 2000).

Another study concentrated on the role of endometrial dysfunction as a sole reason for higher risk of RPL among obese women and it was concluded that obesity promotes endometrial dysfunction by influencing some major protein expression such as beta globulin and transthyretin (Metwally et al 2014). While it has commonly believed that endocrinological and endometrial abnormalities are present in about a quarter of women with unexplained recurrent miscarriage. However, abnormal prolactin, thyroid hormones, and aneuploidy have been ruled out as the main reason behind the increase RPL among obese women (Li et al., 2000; Boots et al., 2014).

This study also found that women with RPL have significantly higher mean value of waist circumference (WC) and waist-hip ratio (WHR). Also Women with a high-risk range of WC were found to have almost 4 fold higher odds RPL compared to those with normal range (table 2). Additionally, Receiver Operator characteristic (ROC) curve showed that WC has considerably higher area under the curve (AUC) and BMI had least area value. Despite the WHO declaration that increased WC poses a relatively higher health risk in obesity compared to BMI, no previous study has shown a clear relationship between RPL and WC. Increased visceral fat in women is a pointer to multiple systemic anomalies that can potentially affect the pregnancy outcome. For instance, in Warming et al., (2003), visceral fat is correlated to the endometrial thickness and free androgen index (FAI) (Nielsen et al., 2007). Visceral at is also associated with increased FSH level and glutathione peroxidase 
activity which leads to accumulation of free radicals and oxidative stress in the reproductive system (Klisic et al., 2018). Leeners et al., (2017) have shown that raised visceral fat in women is a pointer to abnormal serum estrogen or estrogen receptor signaling and either one may have a significant impact on a woman's reproductive health. Women with increased visceral fat tend to have diminished levels of sex hormone-binding globulin and a low level of free 17 beta-estradiol and testosterone ratio signifying androgenicity, owing to the elevated levels of free testosterone (Leenen et al., 1994). Women with higher FAI and higher free testosterone have been shown to have a higher risk of RPL (Okon et al., 1998).

\section{CONCLUSION}

This study has shown that while obesity is a significant risk factor and predictor of RPL among the study participants, measures of visceral adiposity have more predictive power for RPL and may be used to screen those women and risk. High value of WC and WHR May be considered as potential modifiable risk factors of RPL.

This study recommended that clinicians should consider visceral adiposity assessment in predicting risk of RPL. Also, physical therapist should focus more attention to loss of abdominal obesity for those women who engage in weight loss activity in hope of future pregnancies since it is easier to be lost through exercise.

\section{Acknowledgement}

We wish to acknowledge all individuals and organizations who have contributed to the success of this project.

\section{REFERENCE}

Aruna, M., Dasgupta, S., Sirisha, P. V., Andal Bhaskar, S., Tarakeswari, S., Singh, L., \& Reddy, B. M. (2011). Role of androgen receptor CAG repeat polymorphism and Xinactivation in the manifestation of recurrent spontaneous abortions in Indian women. PloS one, 6(3), e17718. https://doi.org/10.1371/journal.pone.0017718

Boots, C. E., Bernardi, L. A., \& Stephenson, M. D. (2014). Frequency of euploid miscarriage is increased in obese women with recurrent early pregnancy loss. Fertility and sterility, 102(2), 455-459.

Boots, C., Stephenson, M.D, (2011). Does obesity increase the risk of miscarriage in spontaneous conception? Seminars in Reproductive Medicine 29:507-513

Cavalcante, M. B., Sarno, M., Peixoto, A. B., Araujo Júnior, E., \& Barini, R. (2019). Obesity and recurrent miscarriage: A systematic review and meta-analysis. The journal of obstetrics and gynaecology research, 45(1), 30-38

Dan Asabe, A. (1991). 'Yan Daba: The "terrorists" of Kano Metropolitan? Kano Studies, Special Issue: Youth \& Health in Kano Today, 3, 85-112.

Du Fossé, N. A., van der Hoorn, M. P., van Lith, J., le Cessie, S., \& Lashley, E. (2020). Advanced paternal age is associated with an increased risk of spontaneous miscarriage: a systematic review and meta-analysis. Human reproduction update, 26(5), 650-669. Https://doi.org/10.1093/humupd/dmaa010

ESHRE Guideline Group on RPL, Bender Atik, R., Christiansen, O. B., Elson, J., Kolte, A. M., Lewis, S., Middeldorp, S., Nelen, W., Peramo, B., Quenby, S., Vermeulen, N., \& Goddijn, M. (2018). ESHRE guideline: recurrent pregnancy loss. Human reproduction open, 2018(2), hoy004. https://doi.org/10.1093/hropen/hoy004 
Ford, H. B., \& Schust, D. J. (2009). Recurrent pregnancy loss: aetiology, diagnosis, and therapy. Reviews in Obstetrics and Gynecology, 2:76-83.

Frick A. P. (2021). Advanced maternal age and adverse pregnancy outcomes. Best practice \& research. Clinical obstetrics \& gynaecology, 70, 92-100.

Hsieh, S. D., \& Yoshinaga, H. (1999). Do people with similar waist circumference share similar health risks irrespective of height?. The Tohoku journal of experimental medicine, 188(1), 55-60. https://doi.org/10.1620/tjem.188.55

Hong Li, Y., \& Marren, A. (2018). Recurrent pregnancy loss: A summary of international evidence-based guidelines and practice. Australian journal of general practice, 47(7), 432-436. https:// doi.org/10.31128/AJGP-01-18-4459

Klisic, A., Kotur-Stevuljevic, J., Kavaric, N., Martinovic, M., \& Matic, M. (2018). The association between follicle stimulating hormone and glutathione peroxidase activity is dependent on abdominal obesity in postmenopausal women. Eating and Weight Disorders-Studies on Anorexia, Bulimia and Obesity, 23(1), 133-141.

Lashen, H., Fear, K., \& Sturdee, D. W. (2004). Obesity is associated with increased risk of first trimester and recurrent miscarriage: matched case-control study. Human reproduction (Oxford, England), 19(7), 1644-1646.

Leenen, R., Van Der Kooy, K., Seidell, J. C., Deurenberg, P., \& Koppeschaar, H. P. (1994). Visceral fat accumulation in relation to sex hormones in obese men and women undergoing weight loss therapy. The Journal of Clinical Endocrinology $\mathcal{E}$ Metabolism, 78(6), 1515-1520.

Leeners B., Geary, N., Tobler, P. N., \& Asarian L. (2017). Ovarian hormones and obesity, Human Reproduction Update. 23: 300-321,

Leeners, B., Geary, N., Tobler, P. N., \& Asarian, L. (2017). Ovarian hormones and obesity. Human reproduction update, 23(3), 300-321. https://doi.org/10.1093/humupd/dmw045

Li, T. C., Spuijbroek, M. D., Tuckerman, E., Anstie, B., Loxley, M., \& Laird, S. (2000). Endocrinological and endometrial factors in recurrent miscarriage. BJOG : an international journal of obstetrics and gynaecology, 107(12), 1471-1479. https://doi.org/10.1111/j.1471-0528.2000.tb11670.x

Lo, W., Rai, R., Hameed, A., Brailsford, S. R., Al-Ghamdi, A. A., \& Regan, L. (2012). The effect of body mass index on the outcome of pregnancy in women with recurrent miscarriage. Journal of family \& community medicine, 19(3), 167-171. https:// doi.org/10.4103/2230-8229.102316

Longo, M., Zatterale, F., Naderi, J., Parrillo, L., Formisano, P., Raciti, G. A., Beguinot, F., \& Miele, C. (2019). Adipose Tissue Dysfunction as Determinant of Obesity-Associated Metabolic Complications. International journal of molecular sciences, 20(9), 2358. https:// doi.org/10.3390/ijms20092358

Metwally, M., Preece, R., Thomas, J., Ledger, W., \& Li, T. C. (2014). A proteomic analysis of the endometrium in obese and overweight women with recurrent miscarriage: preliminary evidence for an endometrial defect. Reproductive Biology and Endocrinology, 12(1), 1-10.

Metwally, M., Saravelos, S. H., Ledger, W. L., \& Li, T. C. (2010). Body mass index and risk of miscarriage in women with recurrent miscarriage. Fertility and sterility, 94(1), 290-295.

Mueller, W. H., Harrist, R. B., Doyle, S. R., \& Labarthe, D. R. (2004). Percentiles of body composition from bioelectrical impedance and body measurements in U.S. adolescents 8-17 years old: Project HeartBeat!. American journal of human biology : the official journal of the Human Biology Council, 16(2), 135-150. https://doi.org/10.1002/ajhb.20002

Nielsen, T. L., Hagen, C., Wraae, K., Brixen, K., Petersen, P. H., Haug, E., ... \& Andersen, M. (2007). Visceral and subcutaneous adipose tissue assessed by magnetic resonance 
imaging in relation to circulating androgens, sex hormone-binding globulin, and luteinizing hormone in young men. The Journal of Clinical Endocrinology $\mathcal{E}$ Metabolism, 92(7), 2696-2705.

Nybo Andersen, A. M., Wohlfahrt, J., Christens, P., Olsen, J., \& Melbye, M. (2000). Maternal age and fetal loss: population based register linkage study. BMJ

(Clinical research ed.), 320(7251), 1708-1712. Https:// doi.org/10.1136/bmj.320.7251.1708

Okon, M. A., Laird, S. M., Tuckerman, E. M., \& Li, T. C. (1998). Serum androgen levels in women who have recurrent miscarriages and their correlation with markers of endometrial function. Fertility and sterility, 69(4), 682-690

Ozawa, N., Ogawa, K., Sasaki, A., Mitsui, M., Wada, S., \& Sago, H. (2019). Maternal age, history of miscarriage, and embryonic/fetal size are associated with cytogenetic results of spontaneous early miscarriages. Journal of assisted reproduction and genetics, 36(4), 749-757. https:// doi.org/10.1007/s10815-019-01415-y

Palmer, B. F., \& Clegg, D. J. (2015). The sexual dimorphism of obesity. Molecular and cellular endocrinology, 402, 113-119. https://doi.org/10.1016/j.mce.2014.11.029

Polotsky, A.J., Hailpern, S.M., Skurnick, J.H., Lo, J.C., Sternfeld, B., Santoro, N, (2011). Association of adolescent obesity and lifetime nulliparity: The Study of Women's Health Across the Nation (SWAN). Fertility and Sterility 93:2004-2011.

Price, G. M., Uauy, R., Breeze, E., Bulpitt, C. J., \& Fletcher, A. E. (2006). Weight, shape, and mortality risk in older persons: elevated waist-hip ratio, not high body mass index, is associated with a greater risk of death1-3. The American Journal of Clinical Nutrition, 84(2), 449-460

Sugiura-Ogasawara M. (2015). Recurrent pregnancy loss and obesity. Best practice \& research. Clinical obstetrics \& gynaecology, 29(4), 489-497. doi: 10.1016/j.bpobgyn.2014.12.001

Tavoli, Z., Mohammadi, M., Tavoli, A., Moini, A., Effatpanah, M., Khedmat, L., \& Montazeri, A. (2018). Quality of life and psychological distress in women with recurrent miscarriage: a comparative study. Health and quality of life outcomes, 16(1), 15.

Warming, L., Ravn, P., \& Christiansen, C. (2003). Visceral fat is more important than peripheral fat for endometrial thickness and bone mass in healthy postmenopausal women. American journal of obstetrics and gynecology, 188(2), 349-353.

Zhu, J. L., Madsen, K. M., Vestergaard, M., Olesen, A. V., Basso, O., \& Olsen, J. (2005). Paternal age and congenital malformations. Human reproduction (Oxford, England), 20(11), 3173-3177. https:// doi.org/10.1093/humrep/dei186 